\title{
Regularities of the quantum spin Hall phase formation in three-dimensional tetradymite-like topological insulator thin films
}

\author{
T. V. Menshchikova, ${ }^{1,2,{ }^{*}}$ O. Yu. Silkina, ${ }^{1}$ I. V. Silkin, ${ }^{1}$ V. M. Kuznetsov, ${ }^{1}$ and E. V. Chulkov ${ }^{2,1,3,4}$ \\ ${ }^{1}$ Tomsk State University, pr. Lenina 36, 634050 Tomsk, Russia \\ ${ }^{2}$ Donostia International Physics Center, P. Manuel de Lardizabal 4, San Sebastián, 20018 Basque Country, Spain \\ ${ }^{3}$ St. Petersburg State University, 198504 St. Petersburg, Russia \\ ${ }^{4}$ Departamento de Fisica de Materiales, Facultad de Ciencias Quimicas, UPV/EHU and Centro de Fisica de Materiales, Centro Mixto \\ CSIC-UPV/EHU, Apartado 1072, 20080 San Sebastian/Donostia, Basque Country, Spain
}

(Received 23 August 2017; published 30 October 2017)

\begin{abstract}
We present first-principles calculations of electronic structure and topological properties of thin films of tetradymite-like binary, ternary, and quaternary compounds. Two-dimensional topological insulators were found, including two septuple layer films of $\mathrm{PbBi}_{2} \mathrm{Te}_{4}$ with large band gap. We demonstrate that the Pb-based thin films inherit $\mathbb{Z}_{2}$ dependence on the film thickness from the "parent" quintuple-layer compounds. An effective way to induce the topological phase transition in the trivial thin films is proposed that does not require substantial changes in the in-plane lattice parameter and surface crystal structure.
\end{abstract}

DOI: 10.1103/PhysRevB.96.165441

\section{INTRODUCTION}

A theoretical prediction and further experimental verification of quantum spin Hall (QSH) effect induced by spinorbit interaction in two-dimensional (2D) insulating systems lead to the identification of a state of quantum mattertwo-dimensional topological insulators (2D TIs) [1-7]. Such systems are characterized by topological invariant $\mathbb{Z}_{2}=1$ and helical gapless edge states protected by time-reversal symmetry from backscattering. As a result, charge carriers at the edges provide dissipationless spin-polarized current [8], making 2D TIs promising for applications in low-powerconsumption electronic devices.

To date, QSH effect has been theoretically predicted in a number of compounds containing heavy elements like Te, $\mathrm{Bi}$, $\mathrm{Pb}, \mathrm{Mo}, \mathrm{Ti}$, and Sn [9-33]. In particular, such compounds are by nature of different types of monolayers $[9,16]$, bilayers $[12,14], 2 \mathrm{D}$ inversion-asymmetric TIs [20], chemically functionalized systems [21,22], heterostructures [33], and others. Despite this successful progress in the theoretical prediction of the QSH systems, most proposed 2D materials have not been fabricated and experimentally verified.

One of the central topics in this field is the search of $2 \mathrm{D}$ TI materials simultaneously combining both sizable band gap and easy fabrication. Promising candidates for realization of the QSH state in 2D systems can be thin films of 3D TIs with layered tetradymite-like crystal structure [26-31,34-37]. Such films can be easily produced by using the advanced technology of nano-ribbon growth method or molecular beam epitaxy. Note that the high-quality samples of thin films of 3D TIs, for instance $\mathrm{Bi}_{2} \mathrm{Se}_{3}[38,39]$ and $\mathrm{Bi}_{2} \mathrm{Te}_{3}[40,41]$, have already been fabricated. Additionally, tetradymite-like layered compounds can form homologous series of ternary and quaternary compounds, including a family of natural minerals [42-44]. As a result, the common factors in the QSH phase formation in such thin films can be found, which have not been investigated yet. Also in such systems, the oscillations of the

*menshikova_t@mail.ru
$\mathbb{Z}_{2}$ topological invariant with an increase of the film thickness can be expected [29-31].

In this paper, we present a detailed investigation of the topological properties of binary, ternary, and quaternarycompound thin films. For the binary-compound thin films, we show the dependence of the topological properties on crystal structure parameters and calculation details. We demonstrate the dependence of the thin film topological properties on the chemical composition, and predict the existence of the QSH phase among $\mathrm{Bi}_{2} \mathrm{Te}_{2} \mathrm{~S}, \mathrm{Bi}_{2} \mathrm{Te}_{2} \mathrm{Se}$, and $\mathrm{Bi}_{2} \mathrm{Te}_{2.4} \mathrm{Se}_{0.6}$ thin films. In the latter case, we find that the type of disordering strongly influences the topological properties. We predict existence of 2D TIs among thin films of Pb-based compounds. Moreover, $\mathrm{Pb}$-based compound thin films are characterized by the same dependence of the $\mathbb{Z}_{2}$ topological invariant on the thickness that is found for "parent" quintuple-layer $(\mathrm{QL})$ compounds. We also demonstrate the effective way of tuning topological properties, which provides an opportunity to induce the QSH state in ultrathin films.

The paper is organized as follows. Section II provides computational details. In Sec. III, we present a study of topological properties depending on the film thickness in binary and ternary compounds. The conclusions are given in Sec. IV.

\section{CALCULATION METHODS}

Electronic structure calculations were carried out using the projector augmented-wave (PAW) [45,46] and full-potential linearized augmented plane wave (FLAPW) methods implemented in the ABINIT [47] and FLEUR [48] code, respectively. The PAW data sets in the ABINIT code were taken from Ref. [49]. The exchange-correlation energy was treated using the generalized gradient approximation (GGA) with PBE functional [50], and local density approximation (LDA) with PW functional [51] for ABINIT, and PZ functional for FLEUR [52]. The Hamiltonian contained scalar relativistic corrections and the spin-orbit coupling was taken into account by the second variation method [53]. The $\mathbb{Z}_{2}$ invariant was obtained 
(a)

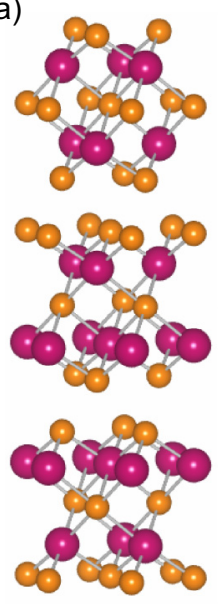

(b)

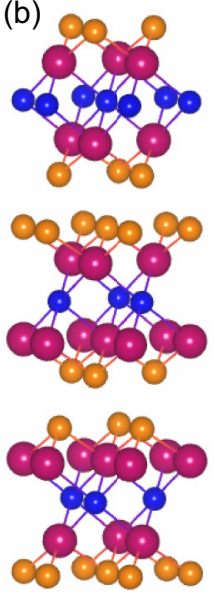

$\mathrm{Bi}_{2} \mathrm{Te}_{3}$ (c)
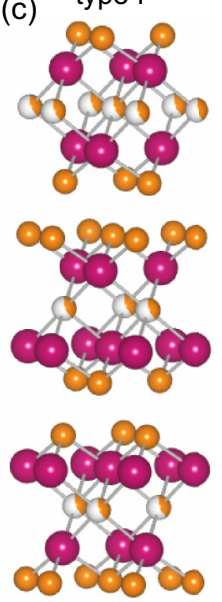

$\mathrm{Bi}_{2} \mathrm{Te}_{2.4} \mathrm{Se}_{0.6}$

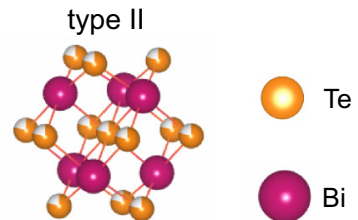

Te

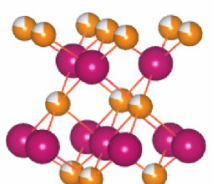

O $\mathrm{s}$
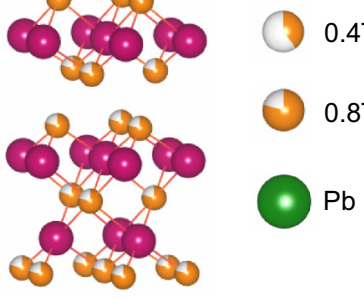

(d)
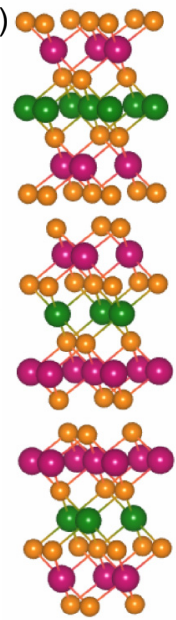

$\mathrm{PbBi}_{2} \mathrm{Te}_{4}$ (e)
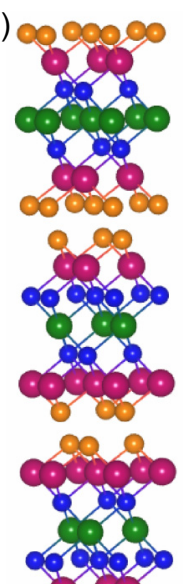

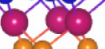

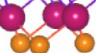

$\mathrm{PbBi}_{2} \mathrm{Te}_{2} \mathrm{~S}_{2}$

FIG. 1. Atomic structure of quintuple-layer binary, ternary (a)-(c), and septuple-layer Pb-based (d), (e) compounds.

from the parity of occupied electronic states at the timereversal invariant points of the $2 \mathrm{D}$ Brillouin zone [54] within both methods.

The electronic structures of thin films were calculated by using various structural parameter sets. The first one includes several sets of experimental crystallographic data. The second set is a structure geometry obtained from optimization of interatomic distances at fixed experimental lattice parameters $a$ and $c$ in the bulk (denoted as "EXPn-opt," $\mathrm{n}$ is a variant of experimental data). In the case of $\mathrm{Bi}_{2} \mathrm{Se}_{3}$, full optimization of the bulk parameters was also done, including cell parameters and interatomic distances (see Supplemental Material (SM) [55] for more details). The last configuration of thin films includes the surface relaxation: the atomic layers in the outermost structural blocks (from vacuum side) were relaxed with inclusion of van der Waals (vdW) correction [56].

To treat the disordered phase, we employed a virtual crystal approximation (VCA) as implemented in the ABINIT code where the averaged ionic pseudopotential of a virtual atom occupying a site in the $\mathrm{A} / \mathrm{B}$ sublattice is defined as a mixture $\mathrm{V}_{\mathrm{VCA}}=\mathrm{xV}_{A}+(1-\mathrm{x}) \mathrm{V}_{B}$ of $\mathrm{A}\left(\mathrm{V}_{A}\right)$ and $\mathrm{B}\left(\mathrm{V}_{B}\right)$ pseudopotentials. In our paper, the role of $A / B$ sublattice plays $\mathrm{Bi} / \mathrm{Sb}$ or $\mathrm{Te} / \mathrm{Se}$ sublattice. In the ABINIT calculations for this case, we used GGA-PBE Hartwigsen-Goedecker-Hutter relativistic norm-conserving pseudopotentials, which include the spin-orbit interaction [57].

\section{RESULTS AND DISCUSSION}

\section{A. Topological properties of quintuple-layer thin films}

The binary compounds we study have a tetradymite-like structure with alternation of weakly bounded vdW forces, QL blocks along the $c$ axis [Fig. 1(a)]. Despite many electronic structure calculations of binary compound thin films performed to date, the topological properties of these thin films are still under debate. For instance, in Ref. [30] the 2D QSH state in some thin films of $\mathrm{Bi}_{2} \mathrm{Se}_{3}$ was shown to exist by both analytical models and first-principles calculations. However, recent GW (GW approximation is expansion of the self-energy $\Sigma$ in terms of the single particle Green's function $G$ and the screened Coulomb interaction W) [26] and full potential ELAPW (extended linearized augmented plane wave method) [31] calculations demonstrated that thin films of $\mathrm{Bi}_{2} \mathrm{Se}_{3}$ are topologically trivial. For this reason, we start with a detailed investigation of the topological properties of thin films $\mathrm{Bi}_{2} \mathrm{Se}_{3}, \mathrm{Bi}_{2} \mathrm{Te}_{3}$, and $\mathrm{Sb}_{2} \mathrm{Te}_{3}$ in dependence on different structure parameters and exchange-correlation energy within PAW and FLAPW methods implemented in the ABINIT and the FLEUR code, respectively (see S1 in the SM [55]).

Summarizing the obtained results, we conclude that the GGA approximation, in general, gives more consistent results compared to those obtained with the LDA. The optimization of the crystal structure can dramatically affect the topological properties. Also, strong sensitivity of these properties to the calculation method was found in the case of $\mathrm{Bi}_{2} \mathrm{Te}_{3}$ ( $\mathrm{S} 1$ in the $\mathrm{SM}$ [55]). The analysis of the obtained results showed that the $\mathrm{Bi}_{2} \mathrm{Se}_{3}$ thin films are trivial. In the case of $\mathrm{Bi}_{2} \mathrm{Te}_{3}$, the thin film topological invariant changes a minimum of twice (Table I), and the $\mathrm{Sb}_{2} \mathrm{Te}_{3}$ thin films are 2D TIs with a thickness of 3 QLs and 4 QLs.

TABLE I. The calculated $\mathbb{Z}_{2}$ topological invariant for quintuplelayer thin films (ABINIT calculations with PBE exchange-correlation functional).

\begin{tabular}{lcccc}
\hline \hline & $1 Q L$ & 2QLs & 3QLs & 4QLs \\
\hline $\mathrm{Bi}_{2} \mathrm{Se}_{3}$ & 0 & 0 & 0 & 0 \\
$\mathrm{Bi}_{2} \mathrm{Te}_{3}$ & 0 & 1 & 0 & 0 \\
$\mathrm{Sb}_{2} \mathrm{Te}_{3}$ & 0 & 0 & 1 & 1 \\
$\mathrm{Bi}_{2} \mathrm{Te}_{2} \mathrm{~S}$ & 0 & 0 & 1 & 1 \\
$\mathrm{Bi}_{2} \mathrm{Te}_{2} \mathrm{Se}$ & 0 & 1 & 1 & 0 \\
$\mathrm{Bi}_{2} \mathrm{Te}_{2.4} \mathrm{Se}_{0.6}$ type I & 0 & 1 & 0 & 0 \\
$\mathrm{Bi}_{2} \mathrm{Te}_{2.4} \mathrm{Se}_{0.6}$ type II & 0 & 1 & 1 & 0 \\
$\mathrm{Sb}_{2} \mathrm{Te}_{2} \mathrm{~S}$ & 0 & 0 & 0 & 0 \\
$\mathrm{Sb}_{2} \mathrm{Te}_{2} \mathrm{Se}$ & 0 & 0 & 0 & 0 \\
\hline \hline
\end{tabular}



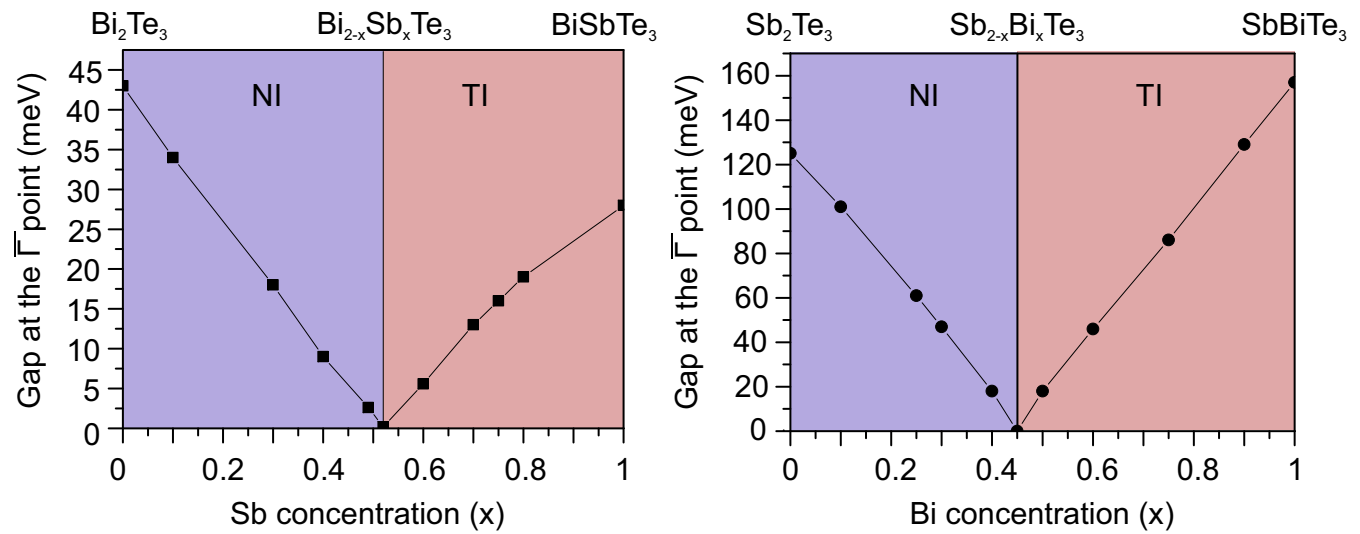

FIG. 2. Phase diagram for alloys of 3 QLs of $\mathrm{Bi}_{2-x} \mathrm{Sb}_{x} \mathrm{Te}_{3}$ and 2 QLs of $\mathrm{Sb}_{2-x} \mathrm{Bi}_{x} \mathrm{Te}_{3}$ (ABINIT calculations with PBE exchange-correlation functional).

The topologically nontrivial properties of thin films are reflected in the inversion of gap edges. A careful analysis shows that the atomic layers near the vdW gaps [the closest$\mathrm{Te}(\mathrm{Se})$, and the second- $\mathrm{Bi}(\mathrm{Sb})]$ are an essential factor responsible for the inversion of the gap edges. It assumes that changing or replacing these layers with other atomic layers can strongly influence the topological properties of thin films. In this context, a relevant modification is replacing of the fourth or fifth layer (near the vdW gap) in the outermost QL blocks by their isoelectronic analog. Such a modification does not cause substantial changes in the in-plane lattice parameter and the surface crystal structure. Note that the mentioned modification will be well pronounced in the case of ultrathin films formed by two or three QLs. Also, these films are more interesting for practical applications due to larger band gaps in comparison with those in the thicker films.

To illustrate this idea, we consider the cases of topologically trivial thin films of binary compounds: $2 \mathrm{QLs}$ of $\mathrm{Bi}_{2} \mathrm{Se}_{3}, 3 \mathrm{QLs}$ of $\mathrm{Bi}_{2} \mathrm{Te}_{3}$, and $2 \mathrm{QLs}$ of $\mathrm{Sb}_{2} \mathrm{Te}_{3}$. First, let us consider simple replacement of the fourth or fifth layer from the vacuum side (near the vdW gap) in the outermost QL blocks by isoelectronic atomic layer without any relaxation. In the case of 2 QLs of $\mathrm{Bi}_{2} \mathrm{Se}_{3}$, the replacement of Se layers forming vdW by Te leads to the topological phase transition. The electronic structure of such a system is characterized by the inverted energy gap and $\mathbb{Z}_{2}=1$. The same effect was found for $3 \mathrm{QLs}$ of $\mathrm{Bi}_{2} \mathrm{Te}_{3}$ upon substitution of Bi layers closest to the vdW spacing by $\mathrm{Sb}$ in the outermost QL blocks. The modification of $2 \mathrm{QLs}$ of $\mathrm{Sb}_{2} \mathrm{Te}_{3}$ by replacement of the fourth $\mathrm{Sb}$ layer by $\mathrm{Bi}$ in the $\mathrm{QL}$ blocks leads to a topologically nontrivial system. Note, similar results were found upon substitution of both $\mathrm{Bi}$ or Sb layers in the outermost QLs of 3 QLs of $\mathrm{Bi}_{2} \mathrm{Te}_{3}$ and 2 QLs of $\mathrm{Sb}_{2} \mathrm{Te}_{3}$, respectively.

Second, as was mentioned above, the optimization of the crystal structure parameters can significantly affect the topological properties. In the case of $2 \mathrm{QLs}$ of $\mathrm{Bi}_{2} \mathrm{Se}_{3}$ with substituted Se layers, the optimization induces a sizable increase of $\mathrm{vdW}$ spacing that is responsible for formation of the topologically trivial phase [32]. In contrast, for modified 3 QLs of $\mathrm{Bi}_{2} \mathrm{Te}_{3}$ and $2 \mathrm{QLs}$ of $\mathrm{Sb}_{2} \mathrm{Te}_{3}$, the optimization does not affect the topological properties, $\mathbb{Z}_{2}=1$, as it happens in the unrelaxed cases.
Additionally, in thin films, the formation of alloy systems like $\mathrm{Bi}_{2-x} \mathrm{Sb}_{x} \mathrm{Te}_{3}$ and $\mathrm{Sb}_{2-x} \mathrm{Bi}_{x} \mathrm{Te}_{3}$ is also possible. To examine the evolution of topological characteristics in such films, let us consider the 3 QLs of $\mathrm{Bi}_{2-x} \mathrm{Sb}_{x} \mathrm{Te}_{3}$ and 2 QLs of $\mathrm{Sb}_{2-x} \mathrm{Bi}_{x} \mathrm{Te}_{3}$ with systematically varying the concentration $\mathrm{x}$. In this context, the topological transition occurs at a certain concentration of $x$ that is characterized by closing the band gap [58]. As seen in Fig. 2, the topological phase transition takes place in $\mathrm{Bi}_{1.48} \mathrm{Sb}_{0.52} \mathrm{Te}_{3}$ and $\mathrm{Sb}_{1.55} \mathrm{Bi}_{0.45} \mathrm{Te}_{3}$ for 3 QLs and 2 QLs, respectively. In general, the dependence of the band gap on concentration is linear for both cases.

Despite the central atomic layer in QL blocks, it makes only a small contribution to the inversion of gap edges, and replacing such a layer in all blocks by another atomic layer can also affect the topological properties due to the changing of the chemical interaction in the system. This situation is realized in the case of ternary tetradymite-like compounds like $\mathrm{Sb}_{2} \mathrm{Te}_{2} \mathrm{~S}$, $\mathrm{Sb}_{2} \mathrm{Te}_{2} \mathrm{Se}$, and $\mathrm{Bi}_{2} \mathrm{Te}_{2} \mathrm{~S}, \mathrm{Bi}_{2} \mathrm{Te}_{2} \mathrm{Se}$ [Fig. 1(b)]. The presence of $\mathrm{S}$ and Se in the center of QLs leads to the increase of chemical bonding within the QLs in comparison with parent binary compounds. The reinforcement of bonds is directly related to higher electronegativity of S and Se in comparison with Te. Moreover, such an increase of chemical bonding is followed by the decrease of the interaction between QL blocks, leading to significant separation of adjacent covalent blocks [59]. In the case of $\mathrm{Sb}_{2} \mathrm{Te}_{2} \mathrm{~S}$ and $\mathrm{Sb}_{2} \mathrm{Te}_{2} \mathrm{Se}$, the vdW gap expands by $\sim 9 \%$ and $7 \%$, respectively, in comparison with $\mathrm{Sb}_{2} \mathrm{Te}_{3}$. As a consequence, all thin films of these compounds are trivial (Table I). The expansion of vdW gap also takes place in ternary compounds $\mathrm{Bi}_{2} \mathrm{Te}_{2} \mathrm{~S}$ and $\mathrm{Bi}_{2} \mathrm{Te}_{2} \mathrm{Se}$. However, the presence of $\mathrm{Bi}$ layers characterized by stronger spin-orbit coupling than $\mathrm{Sb}$ leads to the formation of the QSH phase in thin films of $\mathrm{Bi}_{2} \mathrm{Te}_{2} \mathrm{~S}$ and $\mathrm{Bi}_{2} \mathrm{Te}_{2} \mathrm{Se}$ (Fig. 3). As can be seen from Table I, 3-QL and 4-QL- thin films of $\mathrm{Bi}_{2} \mathrm{Te}_{2} \mathrm{~S}$ are topologically nontrivial. In the case of $\mathrm{Bi}_{2} \mathrm{Te}_{2} \mathrm{Se}$ thin films, the dependence of $\mathbb{Z}_{2}$ on the film thickness is different. The QSH phase was found for smaller thickness of films 2 QLs and 3 QLs. Note, for both compounds, $\mathrm{Bi}_{2} \mathrm{Te}_{2} \mathrm{~S}$ and $\mathrm{Bi}_{2} \mathrm{Te}_{2} \mathrm{Se}$, the electronic spectra of the $2 \mathrm{QL}-4$ QL films are semimetallic.

At the same time, intriguing topological properties can be expected in the case of thin films of recently studied 3D TI $\mathrm{Bi}_{2} \mathrm{Te}_{2.4} \mathrm{Se}_{0.6}[60,61]$. A distinct feature of this compound is 


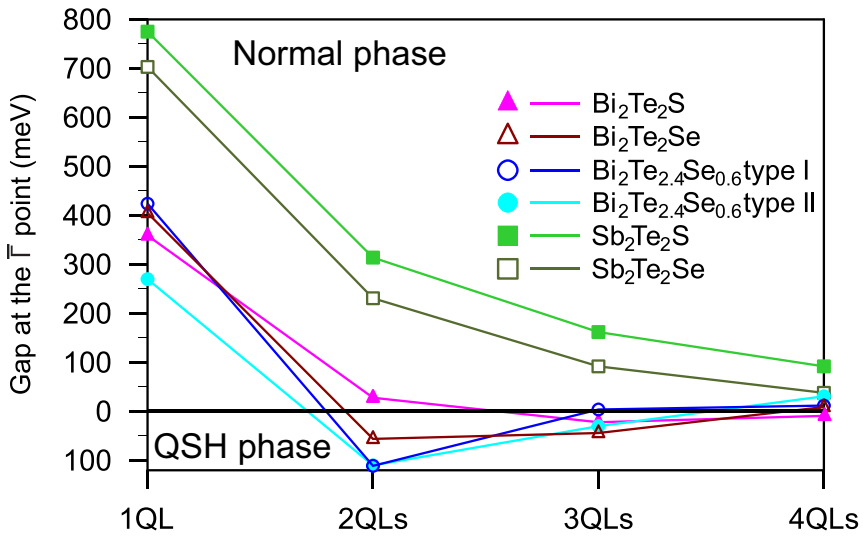

FIG. 3. The dependence of the gap at the $\bar{\Gamma}$ point on the film thickness for ternary quintuple-layer compounds (ABINIT calculations with PBE exchange-correlation functional).

the existence of two possible types of disordering [59,62] [Fig. 1(c)]. The first type of disorder is a substitution of Te atoms in the middle layer of QLs by Se atoms (denoted as type I) [62]. The second one is random distribution of Se atoms in both Te sublattices formed by the central layer and outer Te atoms of QL blocks (denoted as type II) [59]. Since both types of disorder are possible, we consider the topological properties of the $\mathrm{Bi}_{2} \mathrm{Te}_{2.4} \mathrm{Se}_{0.6}$ thin films for type I and type II cases.

Analysis of topological properties of $\mathrm{Bi}_{2} \mathrm{Te}_{2.4} \mathrm{Se}_{0.6}$ thin films with admixture of Se atoms in the middle layer of QLs reveals the same dependence of $\mathbb{Z}_{2}$ on the film thickness as in the case of $\mathrm{Bi}_{2} \mathrm{Te}_{3}$ (Table I). In fact, such a concentration of Se atoms is not enough for tuning thin film topological properties to the $\mathrm{Bi}_{2} \mathrm{Te}_{2} \mathrm{Se}$ case. At the same time, unexpected results were obtained for the random distribution of Se atoms over the Te sublattices (Table I). As can be seen from the Table I, the topologically nontrivial films are 2 QLs and 3 QLs, as in the case of $\mathrm{Bi}_{2} \mathrm{Te}_{2} \mathrm{Se}$. This can be understood from analysis of the crystal structure. The analysis shows the QL blocks of $\mathrm{Bi}_{2} \mathrm{Te}_{2.4} \mathrm{Se}_{0.6}$ (type II) and $\mathrm{Bi}_{2} \mathrm{Te}_{2} \mathrm{Se}$ are chemically close: the value and ratio of the interplanar distances are almost the same for these compounds.

\section{B. Pb-based septuple-layer thin films}

The Pb-based ternary compounds $\left(\mathrm{PbBi}_{2} \mathrm{Se}_{4}, \mathrm{PbBi}_{2} \mathrm{Te}_{4}\right.$, $\mathrm{PbSb}_{2} \mathrm{Te}_{4}$ ) belong to the homologous series of systems $\mathrm{nA}^{I V} \mathrm{~B}^{V I}-\mathrm{mA}_{2}^{V} \mathrm{~B}_{3}^{V I}\left(\mathrm{~A}^{I V}=\mathrm{Ge}, \mathrm{Sn}, \mathrm{Pb} ; \mathrm{A}^{V}=\mathrm{Bi}, \mathrm{Sb} ; \mathrm{B}^{V I}=\right.$ $\mathrm{Te}, \mathrm{Se})$ with $n, m=1$. Their crystal structure is hexagonal $(R \overline{3} \mathrm{~m})$ and the unit cell contains septuple-layer (SL) blocks aligned along the hexagonal $c$ axis [63-65] [Fig. 1(d)]. Quaternary $\mathrm{Pb}$-based compounds $\mathrm{PbBi}_{2} \mathrm{Te}_{2} \mathrm{~S}_{2}$ (aleksite) and $\mathrm{PbBi}_{2} \mathrm{Te}_{2} \mathrm{Se}_{2}$ (poubaite) are members of a natural minerals family and have the same crystal structure as the respective ternary compounds [42,43] [Fig. 1(e)]. As in the case of QL compounds for the Pb-based thin films, we investigate the influence of different structure parameters and calculation methods on their topological properties (see S2 in the SM [55]).

Analysis of the thin film electronic structure shows that all the studied films of $\mathrm{PbBi}_{2} \mathrm{Se}_{4}$ have a band gap, which
TABLE II. The calculated $\mathbb{Z}_{2}$ topological invariant for $\mathrm{Pb}$-based compounds (ABINIT calculations with PBE exchange-correlation functional).

\begin{tabular}{lcccc}
\hline \hline & $1 S L$ & $2 S L s$ & 3SLs & 4SLs \\
\hline $\mathrm{PbBi}_{2} \mathrm{Se}_{4}$ & 0 & 0 & 0 & 0 \\
$\mathrm{PbBi}_{2} \mathrm{Te}_{4}$ & 0 & 1 & 0 & 1 \\
$\mathrm{PbSb}_{2} \mathrm{Te}_{4}$ & 0 & 0 & 1 & 1 \\
$\mathrm{PbBi}_{2} \mathrm{Te}_{2} \mathrm{~S}_{2}$ & 0 & 0 & 1 & 1 \\
$\mathrm{PbBi}_{2} \mathrm{Te}_{2} \mathrm{Se}_{2}$ & 0 & 1 & 1 & 0 \\
\hline \hline
\end{tabular}

monotonically decreases with the increase of the thickness. For $1 \mathrm{SL}$, the gap is indirect, while for the $2 \mathrm{SL}-4 \mathrm{SL}$ films, the gap is direct at the $\bar{\Gamma}$ point. The calculated topological invariant, $\mathbb{Z}_{2}=0$, for all the films of $\mathrm{PbBi}_{2} \mathrm{Se}_{4}$ demonstrates topological triviality of these films (Table II). In the case of $\mathrm{PbBi}_{2} \mathrm{Te}_{4}$, we reveal strong sensitivity of the electronic structure details to the calculation method (see S2 in the SM [55]). However, the topological invariant being identical within both ABINIT and FLEUR calculations demonstrates that 2-SL and 4-SL films are 2D TIs, while $1 \mathrm{SL}$ and $3 \mathrm{SLs}$ are trivial ones. In the case of $\mathrm{PbSb}_{2} \mathrm{Te}_{4}$, the films are characterized by direct (indirect) band gap at thickness of 2 SLs-4 SLs (1 SL). As follows from Table II, the topological $\mathbb{Z}_{2}$ invariant is not zero only for 3 SLs and 4 SLs. It is necessary to note that the topological invariant does not oscillate and changes once. The common feature of the natural mineral thin films is quick closing of the gap at the $\bar{\Gamma}$ point with the increase of their thickness (Fig. 4). $\mathrm{PbBi}_{2} \mathrm{Te}_{2} \mathrm{~S}_{2}$ thin films are topologically nontrivial at $3 \mathrm{SLs}$ and $4 \mathrm{SLs}$, and the $\mathrm{PbBi}_{2} \mathrm{Te}_{2} \mathrm{Se}_{2}$ ones-at 2 SLs and 3 SLs (Table II and Fig. 4).

Summarizing the above-mentioned results, one can note that in the case of $\mathrm{Pb}$-based compounds, the behavior of $\mathbb{Z}_{2}$ topological invariant is the same as for the parent QL compounds. In particular, thin films of $\mathrm{PbBi}_{2} \mathrm{Se}_{4}$ are topologically trivial, as for $\mathrm{Bi}_{2} \mathrm{Se}_{3}$. In the case of $\mathrm{PbBi}_{2} \mathrm{Te}_{4}$, the $\mathbb{Z}_{2}$ topological invariant changes a minimum of twice, as in the case of $\mathrm{Bi}_{2} \mathrm{Te}_{3} . \mathrm{Sb}_{2} \mathrm{Te}_{3}$ and $\mathrm{PbSb}_{2} \mathrm{Te}_{4}$ thin films have a similar $\mathbb{Z}_{2}$ topological invariant, which changes only once. The topological invariant of the $\mathrm{PbBi}_{2} \mathrm{Te}_{2} \mathrm{~S}_{2}$ and $\mathrm{PbBi}_{2} \mathrm{Te}_{2} \mathrm{Se}_{2}$ thin

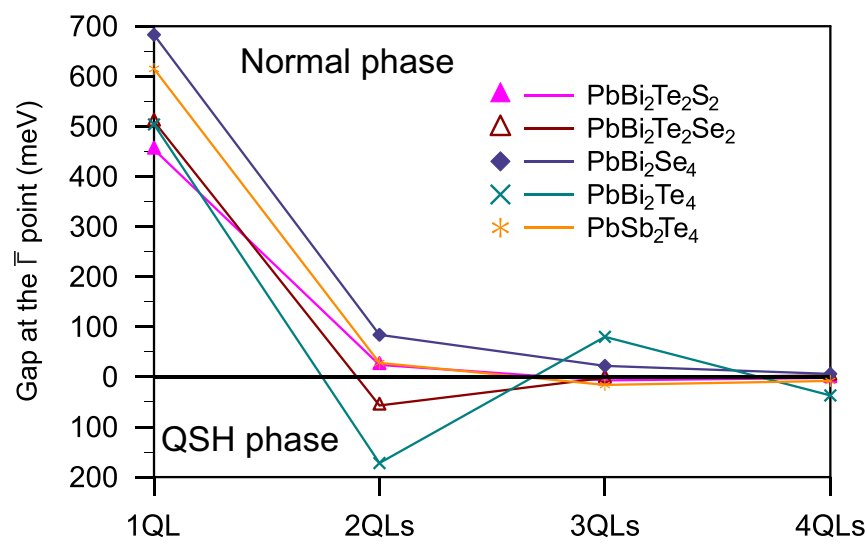

FIG. 4. The dependence of the gap at the $\bar{\Gamma}$ point on the film thickness for Pb-based compounds (ABINIT calculations with PBE exchange-correlation functional). 
films is identical to the one obtained for $\mathrm{Bi}_{2} \mathrm{Te}_{2} \mathrm{~S}$ and $\mathrm{Bi}_{2} \mathrm{Te}_{2} \mathrm{Se}$ thin films, respectively. Such inheritance of the topological properties in $\mathrm{Pb}$-based compounds is directly connected to their crystal structure and peculiarities of the gap edges inversion. The crystal structure of $\mathrm{Pb}$-based compounds can be represented as QLs of binary $\left(\mathrm{Bi}_{2} \mathrm{Se}_{3}, \mathrm{Bi}_{2} \mathrm{Te}_{3}, \mathrm{Sb}_{2} \mathrm{Te}_{3}\right)$ or ternary $\left(\mathrm{Bi}_{2} \mathrm{Te}_{2} \mathrm{Se}, \mathrm{Bi}_{2} \mathrm{Te}_{2} \mathrm{~S}\right)$ compounds with $\mathrm{PbTe}, \mathrm{PbSe}$, or $\mathrm{PbS}$ bilayer inserted into the center of the QL blocks. At the same time, the analysis of the thin film electronic structure shows that the main role in the inversion of the gap edges plays the states formed by atomic layers near the vdW gaps. The central layer states are not involved in the inversion as in the case of QL compounds. In such a way, incorporation of the $\mathrm{PbTe}(\mathrm{PbSe}$ or $\mathrm{PbS})$ bilayer into the QLs center does not change much the orbital composition of the gap edges in comparison with the case of the parent QL compounds. Moreover, the increase of the number of such bilayer does not change the situation either which is corroborated by the results of the Ref. [29] devoted to the study of topological properties of nine-layer $\mathrm{Pb}$-containing ternary compound thin films.

\section{SUMMARY AND CONCLUSION}

In summary, we have studied the electronic structure and topological properties of thin films of binary, ternary, and quaternary compounds. In the example of binary compounds, we have demonstrated the GGA approximation gives more consistent results for electronic structure in comparison with the LDA. Within both ABINIT and FLEUR calculations, we have found the dependence of topological properties on the crystal structure parameters. The full correspondence between these methods was achieved at optimized parameters, including surface relaxation. For the system under debate, $\mathrm{Bi}_{2} \mathrm{Se}_{3}$, all films (1 QL-4 QLs) were found to be topologically trivial within both ABINIT and FLEUR calculations.

We have shown that in ternary QL thin films, the topological properties substantially change in comparison with isostructural binary systems. In particular, all $\mathrm{Sb}_{2} \mathrm{Te}_{2} \mathrm{~S}$ and $\mathrm{Sb}_{2} \mathrm{Te}_{2} \mathrm{Se}$ thin films are topologically trivial. Such a situation is directly related to huge vdW gaps caused by weakening of the interaction between QL blocks. This is caused by the increase of chemical bonding within the QLs due to the presence of the $\mathrm{S}$ and Se atoms, which possess higher electronegativity and smaller size in comparison with Te.

We have predicted 2D TIs among $\mathrm{Pb}$-based thin films characterized by the same dependence of $\mathbb{Z}_{2}$ on the film thickness as the parent QL compounds. Such inheritance of the topological properties is directly related to the crystal structure and the fact that the atomic layers located close to the vdW gap are responsible for topological properties of these films. None of the studied ultrathin $\mathrm{PbBi}_{2} \mathrm{Se}_{4}$ (0001) films are 2D topological insulators. We predict the QSH phase for $\mathrm{PbSb}_{2} \mathrm{Te}_{4}$ and $\mathrm{PbBi}_{2} \mathrm{Te}_{4}$ films. In the latter case, the films formed by the odd number of SL blocks (one and three) are not 2D topological insulators, while the films formed by the even number of blocks (two and four) are topological insulators. The $\mathrm{PbBi}_{2} \mathrm{Te}_{4}$ (0001) film with 2 SL thickness is most promising for the experimental observation of topological edge electronic states because it is characterized by the largest gap. In the case of natural mineral thin films, the dependence of the topological properties is identical to the parent ternary compounds.

Finally, we have proposed an effective way to induce the topological phase transition in the trivial thin films. This way, the replacement of the layers closest to the vdW gap in the outermost structural blocks by isoelectronic atomic analog does not require substantial changes in the in-plane lattice parameter and surface crystal structure. This method will be very efficient in the case of ultrathin films formed by two or three structural blocks.

It is necessary to notice that results may depend on the exchange-correlation functional used. Therefore, all results and conclusions in this paper are valid for the PBE exchangecorrelation functional we used in the calculations. We hope that our findings help to shed light on the topological properties of 2D systems and will be useful for experimental investigations.

\section{ACKNOWLEDGMENTS}

This paper is supported by Tomsk State University competitiveness improvement programme (Project No. 8.1.01.2017), the Saint Petersburg State University Project No. 15.61.202.2015, the Ministry of Education and Science of the Russian Federation within the framework of the governmental program Megagrants (State Task No. 3.8716.2017/П220 or 3.8716.2017/9.10), the University of the Basque Country (Grants No. GIC07IT36607 and No. IT-756-13), the Spanish Ministry of Science and Innovation (Grants No. FIS201348286-C02-02-P, No. FIS2013-48286-C02-01-P, and No. FIS2016-75862-P). Calculations were performed at the SKIFCyberia supercomputer of Tomsk State University and at the Research park of St. Petersburg State University Computing Center (Russian Federation).
[1] C. L. Kane and E. J. Mele, Phys. Rev. Lett. 95, 226801 (2005).

[2] C. L. Kane and E. J. Mele, Phys. Rev. Lett. 95, 146802 (2005).

[3] B. A. Bernevig and S.-C. Zhang, Phys. Rev. Lett. 96, 106802 (2006).

[4] S. Murakami, Phys. Rev. Lett. 97, 236805 (2006).

[5] M. Z. Hasan and C. L. Kane, Rev. Mod. Phys. 82, 3045 (2010).

[6] M. Konig, S. Wiedmann, C. Brune, A. Roth, H. Buhmann, L. W. Molenkamp, X. L. Qi, and S. C. Zhang, Science 318, 766 (2007).

[7] G. Bihlmayer, Y. M. Koroteev, T. V. Menshchikova, E. V. Chulkov, and S. Blügel, Topological Insulators: Fundamentals and Perspectives (Wiley-VCH Verlag GmbH \& Co. KGaA, Weinheim, Germany, 2015), Part II, Chap. 5.

[8] V. N. Men'shov, I. A. Shvets, V. V. Tugushev, and E. V. Chulkov, Phys. Rev. B 96, 075302 (2017).

[9] Y. Ma, L. Kou, X. Li, Y. Dai, S. C. Smith, and T. Heine, Phys. Rev. B 92, 085427 (2015).

[10] S. M. Nie, Z. Song, H. Weng, and Z. Fang, Phys. Rev. B 91, 235434 (2015).

[11] X. Qian, J. Liu, L. Fu, and J. Li, Science 346, 1344 (2014).

[12] F.-C. Chuang, L.-Z. Yao, Z.-Q. Huang, Y.-T. Liu, C.-H. Hsu, T. Das, H. Lin, and A. Bansil, Nano Lett. 14, 2505 (2014). 
[13] W. Luo and H. J. Xiang, Nano Lett. 15, 3230 (2015).

[14] Y. D. Ma, Y. Dai, L. Z. Kou, T. Frauenheim, and T. Heine, Nano Lett. 15, 1083 (2015).

[15] M. Zhou, W. Ming, Z. Liu, Z. F. Wang, P. Li, and F. Liu, Proc. Natl. Acad. Sci. USA 111, 14378 (2014).

[16] Z. G. Song, C. C. Liu, J. B. Yang, J. Z. Han, B. T. Fu, M. Ye, Y. C. Yang, Q. Niu, J. Lu, and Y. G. Yao, NPG Asia Mater. 6, e147 (2014).

[17] Y. D. Ma, L. Z. Kou, X. Li, Y. Dai, and T. Heine, NPG Asia Mater. 8, e264 (2016).

[18] J. J. Zhou, W. X. Feng, C.-C. Liu, S. Guan, and Y. G. Yao, Nano Lett. 14, 4767 (2014).

[19] H. Weng, X. Dai, and Z. Fang, Phys. Rev. X 4, 011002 (2014).

[20] Y. Ma, L. Kou, A. Du, and T. Heine, Nano Res. 8, 3412 (2015).

[21] C. Si, J. Liu, Y. Xu, J. Wu, B.-L. Gu, and W. Duan, Phys. Rev. B 89, 115429 (2014).

[22] Y. Xu, B. Yan, H.-J. Zhang, J. Wang, G. Xu, P. Tang, W. Duan, and S.-C. Zhang, Phys. Rev. Lett. 111, 136804 (2013).

[23] Z. Zhu, Y. Cheng, and U. Schwingenschlögl, Phys. Rev. Lett. 110, 077202 (2013).

[24] Z. F. Wang, L. Chen, and F. Liu, Nano Lett. 14, 2879 (2014).

[25] L. Zhou, L. Kou, Y. Sun, C. Felser, F. Hu, G. Shan, S. C. Smith, B. Yan, and T. Frauenheim, Nano Lett. 15, 7867 (2015).

[26] T. Förster, P. Krüger, and M. Rohlfing, Phys. Rev. B 92, 201404 (2015).

[27] K. Minsung, H. K. Choong, K. Heung-Sik, and I. Jisoon, Proc. Natl. Acad. Sci. USA 109, 671 (2012).

[28] B. Singh, H. Lin, R. Prasad, and A. Bansil, Phys. Rev. B 88, 195147 (2013).

[29] I. V. Silkin, Y. M. Koroteev, S. V. Eremeev, G. Bihlmayer, and E. V. Chulkov, JETP Lett. 94, 217 (2011).

[30] C.-X. Liu, H.-J. Zhang, B. Yan, X.-L. Qi, T. Frauenheim, X. Dai, Z. Fang, and S.-C. Zhang, Phys. Rev. B 81, 041307 (2010).

[31] I. A. Nechaev and E. E. Krasovskii, Phys. Rev. B 94, 201410 (2016).

[32] I. A. Nechaev, S. V. Eremeev, E. E. Krasovskii, P. M. Echenique, and E. V. Chulkov, Sci. Rep. 7, 43666 (2017).

[33] L. Kou, S.-C. Wu, C. Felser, T. Frauenheim, C. Chen, and B. Yan, ACS Nano 8, 10448 (2014).

[34] W.-Y. Shan, H.-Z. Lu, and S.-Q. Shen, NJP 12, 043048 (2010).

[35] H.-Z. Lu, W.-Y. Shan, W. Yao, Q. Niu, and S.-Q. Shen, Phys. Rev. B 81, 115407 (2010).

[36] H. Li, L. Sheng, D. N. Sheng, and D. Y. Xing, Phys. Rev. B 82, 165104 (2010).

[37] G. Landolt, S. Schreyeck, S. V. Eremeev, B. Slomski, S. Muff, J. Osterwalder, E. V. Chulkov, C. Gould, G. Karczewski, K. Brunner, H. Buhmann, L. W. Molenkamp, and J. H. Dil, Phys. Rev. Lett. 112, 057601 (2014).

[38] G. Zhang, H. Qin, J. Teng, J. Guo, Q. Guo, X. Dai, Z. Fang, and K. Wu, Appl. Phys. Lett. 95, 053114 (2009).

[39] M. Neupane, A. Richardella, J. Sánchez-Barriga, S. Xu, N. Alidoust, I. Belopolski, C. Liu, D. Bian, G. Zhang, D. Marchenko, A. Varykhalov, O. Rader, M. Leandersson, T. Balasubramanian, T.-R. Chang, H.-T. Jeng, S. Basak, H. Lin, A. Bansil, N. Samarth, and M. Z. Hasan, Nat. Commun. 5, 3841 (2014).

[40] Y.-Y. Li, G. Wang, X.-G. Zhu, M.-H. Liu, C. Ye, X. Chen, Y.-Y. Wang, K. He, L.-L. Wang, X.-C. Ma, H.-J. Zhang, X. Dai, Z. Fang, X.-C. Xie, Y. Liu, X.-L. Qi, J.-F. Jia, S.-C. Zhang, and Q.-K. Xue, Adv. Mater. 22, 4002 (2010).
[41] S. Borisova, J. Krumrain, M. Luysberg, G. Mussler, and D. Grützmacher, Cryst. Growth Des. 12, 6098 (2012).

[42] Z. Johan, P. Picot, and F. Ruhlmann, Canad. Miner. 25, 625 (1987).

[43] H. Liu and L. L. Y. Chang, Amer. Miner. 79, 1159 (1994).

[44] I. V. Silkin, T. V. Menshchikova, M. M. Otrokov, S. V. Eremeev, Y. M. Koroteev, M. G. Vergniory, V. M. Kuznetsov, and E. V. Chulkov, JETP Lett. 96, 322 (2012).

[45] P. E. Blöchl, Phys. Rev. B 50, 17953 (1994).

[46] G. Kresse and D. Joubert, Phys. Rev. B 59, 1758 (1999).

[47] X. Gonze, B. Amadon, P.-M. Anglade, J.-M. Beuken, F. Bottin, P. Boulanger, F. Bruneval, D. Caliste, R. Caracas, M. Côté, T. Deutsch, L. Genovese, P. Ghosez, M. Giantomassi, S Goedecker, D. R. Hamann, P. Hermet, F. Jollet, G. Jomard, S. Leroux, M. Mancini, S. Mazevet, M. J. T. Oliveira, G. Onida, Y. Pouillon, T. Rangel, G.-M. Rignanese, D. Sangalli, R. Shaltaf, M. Torrent, M. J. Verstraete, G. Zerah, and J. W. Zwanziger, Comput. Phys. Commun. 180, 2582 (2009).

[48] http://www.flapw.de.

[49] F. Jollet, M. Torrent, and N. Holzwarth, Comp. Physics Comm. 185, 1246 (2014).

[50] J. P. Perdew, K. Burke, and M. Ernzerhof, Phys. Rev. Lett. 77, 3865 (1996).

[51] S. Goedecker, M. Teter, and J. Hutter, Phys. Rev. B 54, 1703 (1996).

[52] J. P. Perdew and A. Zunger, Phys. Rev. B 23, 5048 (1981).

[53] D. D. Koelling and B. N. Harmon, J. Phys. C: Solid State Phys 10, 3107 (1977).

[54] L. Fu and C. L. Kane, Phys. Rev. B 76, 045302 (2007).

[55] See Supplemental Material at http://link.aps.org/supplemental/ 10.1103/PhysRevB.96.165441 for detailed investigation of the topological properties of binary and ternary $\mathrm{Pb}$-based compound thin films in dependence on different structure parameters and exchange-correlation energy within PAW and FLAPW methods implemented in the ABINIT and the FLEUR code, respectively.

[56] S. Grimme, J. Antony, S. Ehrlich, and H. Krieg, J. Chem. Phys. 132, 154104 (2010).

[57] M. Krack, Theor. Chem. Acc. 114, 145 (2005).

[58] S. Murakami, NJP 9, 356 (2007).

[59] M. Gharsallah, F. Serrano-Sanchez, N. M. Nemes, J. L. Martinez, and J. A. Alonso, Nanoscale Res. Lett. 12, 47 (2017).

[60] A. M. Shikin, I. I. Klimovskikh, S. V. Eremeev, A. A. Rybkina, M. V. Rusinova, A. G. Rybkin, E. V. Zhizhin, J. Sánchez-Barriga, A. Varykhalov, I. P. Rusinov, E. V. Chulkov, K. A. Kokh, V. A. Golyashov, V. Kamyshlov, and O. E. Tereshchenko, Phys. Rev. B 89, 125416 (2014).

[61] I. I. Klimovskikh, D. Sostina, A. Petukhov, A. G. Rybkin, S. V. Eremeev, E. V. Chulkov, O. E. Tereshchenko, K. A. Kokh, and A. M. Shikin, Sci. Rep. 7, 45797 (2017).

[62] Q. Lognoné, F. Gascoin, O. I. Lebedev, L. Lutterotti, S. Gascoin, and D. Chateigner, J. Amer. Ceram. Soc. 97, 2038 (2014).

[63] K. A. Agaev and S. A. Shelimova, Kristallogr. 13(2), 258 (1968).

[64] T. B. Zhukova and A. I. Zaslavskii, Sov. Phys. Crystallogr. 16, 796 (1972).

[65] L. E. Shelimova, O. G. Karpinskii, T. E. Svechnikova, E. S. Avilov, M. A. Kretova, and V. S. Zemskov, Inorg. Mater. 40, 1264 (2004). 